\title{
Sustentabilidade na última milha da distribuição postal em áreas urbanas mediante adoção de triciclos elétricos
}

\section{Cintia Machado de Oliveira ${ }^{1}$, Renata Albergaria de Mello Bandeira², George Vasconcelos Góes ${ }^{3}$, Daniel Neves Schmitz Gonçalves" ${ }^{4}$, Marcio de Almeida D'Agosto ${ }^{5}$}

1UFRJ/COPPE/Programa de Engenharia de Transportes, Brasil, cintia.machado.oliveira.1@gmail.com 2Instituto Militar de Engenharia, UFRJ/COPPE/Programa de Engenharia de Transportes, Brasil, re.albergaria@gmail.com 3Universidade Federal do Rio de Janeiro/COPPE/Programa de Engenharia de Transportes, Brasil, george.vasconcelos@outlook.com ${ }^{4}$ Universidade Federal do Rio de Janeiro/COPPE/Programa de Engenharia de Transportes, Brasil, daniel.schmitz.jf@gmail.com ${ }^{5}$ Universidade Federal do Rio de Janeiro/COPPE/Programa de Engenharia de Transportes, Brasil, dagosto@pet.ufrj.coppe.br

\section{Recebido:}

21 de novembro de 2017

Aceito para publicação:

7 de junho de 2018

Publicado:

4 de novembro de 2018

Editor de área:

Renato Lima

\section{Palavras-chaves:}

Veículo Elétrico,

Triciclo,

Sustentabilidade.

\section{Keywords:}

Electric Vehicle,

Tricycle,

Sustainability.

DOI:10.14295/transportes.v26i3.1525

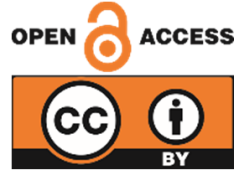

\section{RESUMO}

A demanda por serviços de transporte em todo o mundo é um fenômeno que cresce à medida em que o processo de urbanização avança. No entanto, o excessivo uso de veículos acentua os problemas relacionados às entregas de última milha e à emissão de poluentes atmosféricos locais. $\mathrm{O}$ objetivo deste estudo é avaliar a sustentabilidade alcançada por meio da utilização de triciclos elétricos na distribuição postal na cidade do Rio de Janeiro. Identificou-se a redução de $27,86 \%$ do custo total de transportes. Ademais, observou-se reduções mensais de emissões de poluentes atmosféricos por rota de $10,11 \mathrm{~g}$ de CO; $32,78 \mathrm{~g}$ de NOX; 0,02 $\mathrm{g}$ de RCHO; 0,7 g de NMHC e 0,33 g de MP, enquanto que as reduções mensais de GEE por rota foram de 21,01 kg de CO2eq. Com relação aos aspectos sociais, a frequência cardíaca mostrou-se constante durante toda a operação, enquanto, na operação de distribuição tradicional intermodal, verificou-se uma maior variação durante a operação de entrega.

\section{ABSTRACT}

The demand for transport services around the world is a phenomenon that is directly related to the urbanization process progresses. However, the intensive use of vehicles accentuates problems related to the last mile deliveries and pollutant emissions. This study aims to assess the sustainability obtained using electric tricycles in the postal delivery of Rio de Janeiro. Results indicates a $27.86 \%$ reduction in total transport costs, as well as reductions in monthly emissions of atmospheric pollutants per route by $10.11 \mathrm{~g}$ of $\mathrm{CO} ; 32.78 \mathrm{~g}$ of $\mathrm{NO}_{\mathrm{x}} ; 0.02 \mathrm{~g}$ of $\mathrm{RCHO} ; 0.7 \mathrm{~g} \mathrm{NMHC}$; and $0.33 \mathrm{~g} \mathrm{MP}$, while monthly reductions of $\mathrm{CO}_{2}$ eq emissions per route are $21.01 \mathrm{~kg}$. Regarding the social aspects, the heart rate of the postman is constant throughout the operation, while, in the traditional intermodal distribution, there was a greater variation of the heart rate during the entire delivery process.

\section{INTRODUÇÃO}

De acordo com estimativas das Nações Unidas, cerca de 70\% da população mundial, até 2050, viverá em áreas urbanas (ONU, 2013), o que demonstra uma tendência de crescimento para a demanda dos serviços de transporte em todo o mundo. Adicionalmente, a atividade de transporte, para a distribuição de bens e serviços em áreas urbanas, é responsável por promover impactos sociais, ambientais e econômicos, sobretudo em relação à intensificação dos congestionamentos de tráfego, à emissão de poluentes atmosféricos, poluição sonora e gases do efeito 
estufa (GEE), principalmente o dióxido de carbono $\left(\mathrm{CO}_{2}\right)$, além de promover o aumento dos custos e diminuir a segurança da população, em função do aumento do número de acidentes (Mckinnon et al., 2010; Oliveira et al., 2015).

Os efeitos dessa problemática são potencializados nas operações que envolvem a última milha (last mile), pois é nesta fase em que se evidencia a maior participação dos custos de uma cadeia logística (Roumboutsos et al., 2014). Assim, fabricantes, transportadores e operadores logísticos são instigados a trabalhar a um custo reduzido, porém mantendo o nível de serviço. Esses agentes, portanto, buscam soluções por meio de uma maior cooperação e integração de suas operações, utilizando seus recursos de forma mais eficiente (Steadi e Seifi et al., 2014). Ao mesmo tempo, as cidades precisam avançar em suas transformações e permitir a exploração inovadora de formas de operação do Transporte Urbano de Carga (TUC), sobretudo no que diz respeito ao desenvolvimento de novas tecnologias (Bjorklund e Gustafsson, 2015).

Um estudo realizado na China por Cherry et al. (2009) demonstra que há uma grande busca por estratégias de distribuição urbana de carga, em last mile, não poluentes. Neste cenário, a tecnologia de triciclos elétricos tem sido considerada uma solução alternativa e sustentável de mobilidade urbana, tendo em vista que atende aos anseios de sustentabilidade do mundo contemporâneo, frente à piora da qualidade do ar das cidades, oriunda do uso intenso dos veículos com motor a combustão interna e dos combustíveis fósseis (Cherry et al., 2009). Deste modo, este estudo tem como objetivo avaliar a sustentabilidade, por meio da utilização do triciclo elétrico como alternativa para a distribuição postal em last mile, por meio de um estudo de caso de uma empresa pública, que atua neste segmento, na cidade do Rio de Janeiro, Brasil.

A partir desta introdução, este trabalho se divide em quatro seções. Na seção dois apresentase uma revisão bibliográfica acerca das formas de operação e as tecnologias utilizadas no TUC para distribuição em last mile, em todo o mundo. A metodologia utilizada para o levantamento de dados, bem como a realização da avaliação econômica, ambiental e do nível de serviço oferecido pela empresa, é apresentada na seção três. Na seção quatro, apresentam-se os resultados encontrados e suas respectivas análises. Finalmente, na seção cinco encontram-se as conclusões, limitações e sugestões de aprimoramento deste trabalho.

\section{REVISÃO BIBLIOGRÁFICA}

Com o objetivo de verificar a relevância do estudo de caso que está sendo avaliado neste artigo, optou-se por realizar uma Revisão Bibliográfica Sistemática. A identificação dos artigos foi realizada em duas bases de indexações (ISI Web of Science e SCOPUS) e em cinco bases de dados (EBSCO, Science Direct, DOAJ, Springer Link e Compendex) de modo atender à recomendação de Thomé et al. (2006) de se utilizar mais de duas bases de dados para garantir a identificação de uma maior diversidade de trabalhos.

De forma inicial, foram identificados 157 artigos, que, após a eliminação de artigos duplicados (uma vez que a busca foi realizada em mais de uma base de indexação), resultou em uma lista de 125 publicações, cuja leitura dos resumos foi realizada por pelo menos dois autores, conforme recomendado por Thomé et al. (2016). 0 processo de seleção foi iterativo, de modo a buscar a concordância entre os pesquisadores, sendo selecionados 46 artigos para a leitura completa. Dentre esses, 26 estudos foram inclusos para o desenvolvimento da RBS.

Dos trabalhos inclusos na RBS, 42\% apontaram a utilização de bicicleta/triciclo como melhor alternativa para entregas urbanas de last mile. Destes, no que diz respeito à energia utilizada, $64 \%$ apontaram a energia elétrica e $36 \%$ a propulsão humana. Na outra vertente, os veículos 
do tipo comerciais leves, que se tratam dos veículos com peso bruto total (PBT) inferior a 3,5 toneladas, foram considerados em $58 \%$ dos estudos, dentre os quais, no que diz respeito à fonte de energia utilizada, 73\% apontou a energia elétrica e 33\% apontou outras fontes de energia (diesel de petróleo, metano e biodiesel). Ainda, ressalta-se que um dos estudos considerou a utilização de um veículo do tipo comercial leve, não tripulado, ou seja, veículo autônomo, movido à energia elétrica. Outro estudo apontou a utilização de pequenos carros, bicicleta, triciclo e motocicleta em conjunto aos veículos do tipo comercial leve (todos elétricos), como alternativa para entrega de last mile.

Segundo Schoemaker et al. (2006), o número de veículos do tipo comercial leve, na Europa, aumentou 15\%, entre 1990 e 2003, enquanto o número de veículos com PBT superior a 3,5 toneladas apresentou um aumento de $6.6 \%$, neste mesmo período. Isto reflete a tendência, desde então, do aumento de veículos do tipo comercial leve (em especial vans) no transporte urbano de cargas. Adicionalmente, de acordo com Dablanc (2009), o TUC é ineficiente na maioria das cidades, incluindo aquelas de países desenvolvidos, com baixas taxas de ocupação. Por exemplo, pode-se citar a cidade Londres, onde as taxas de ocupação desses tipos de veículos variavam entre 40 e 60\% em 2006 (Schoemaker et al., 2006). Tal prática também vem sendo aplicada em países em desenvolvimento, como por exemplo o Brasil, onde verificou-se a utilização de triciclos por empresas como Coca-Cola (Fernandes, 2015a) e Correios (Oliveira, 2015), que buscam contornar os efeitos dos congestionamentos do tráfego, das restrições de circulação impostas pelas autoridades nas cidades, empregando práticas sustentáveis do ponto de vista socioambiental que garantam a manutenção ou a melhora do nível de serviço oferecido.

Dessa forma, tais fatos podem justificar a tendência de utilização de veículos ainda menores, tais como triciclos e bicicletas, conforme verificado nesta RBS. Infere-se, portanto que há uma tendência apontada pela literatura especializada na redução do tamanho dos veículos utilizados para entregas urbanas de last mile, com a adoção de bicicletas, triciclos e veículos do tipo comercial leve.

Em referência ao tipo de energia utilizada pelos veículos, considerando todos 26 artigos inclusos na RBS, verificou-se que 69\% abordam veículos movidos a energia elétrica, o que demonstra seu potencial de aplicação na atividade do TUC, sobretudo na distribuição de last mile. Os outros tipos de energia empregados nas aplicações analisadas foram: metano, biodiesel, diesel de petróleo (23\%) e propulsão humana (8\%).

Os estudos apontaram a redução do consumo de energia, redução no tempo de entrega e redução dos congestionamentos. Os benefícios ambientais concentraram-se na redução da emissão de $\mathrm{CO}_{2}$, poluentes atmosféricos e poluição sonora. Quanto aos benefícios sociais, podese destacar a geração de empregos e melhoria na qualidade de vida das pessoas. É oportuno ressaltar que o estudo de Sarma et al. (2014) apontou os problemas de saúde que podem ser acarretados em função da utilização da bicicleta (propulsão humana) e o excesso de peso da carga (195 kg).

Verificou-se que a velocidade média das bicicletas/triciclos variou de 2 a $6 \mathrm{~km} / \mathrm{h}$ (Sarma et al., 2014), enquanto o veículo comercial leve chegou a $25 \mathrm{~km} / \mathrm{h}$ (Gruber et al., 2014). Com relação às restrições de peso, Gruber et al. (2014) apontam $100 \mathrm{~kg}$ para carregamentos em bicicletas elétricas na Alemanha. Já o estudo realizado por Schiliwa et al. (2015) aponta que há uma limitação de peso de até $60 \mathrm{~kg}$ para carregamentos em bicicletas eletro-assistidas na Inglaterra. No entanto, Schier et al. (2016), em sua pesquisa sobre a distribuição urbana de last mile, consideram que bicicletas e triciclos eletro-assistidos podem transportar entre 50 e $250 \mathrm{~kg}$ de 
carga. No caso analisado por Sarma et al. (2014), em Nova Déli, são transportados até $195 \mathrm{~kg}$ de carregamentos por bicicletas movidas à propulsão humana.

\section{MÉTODO}

A distribuição tradicional intermodal (DTI) ocorre, atualmente, por meio de caminhada a pé, a utilização do ônibus convencional e o apoio operacional de um veículo comercial leve com motor à combustão. 0 conhecimento da operação, ou seja, dados operacionais foram obtidos com base em visitas técnicas à empresa.

Deste modo, propõe-se a alternativa de distribuição postal com o apoio de Triciclo Elétrico (DTE). Para avaliar a distribuição DTE, realizou-se os testes nos bairros do Leme e Copacabana na cidade do Rio de Janeiro, Brasil. Estes bairros apresentam uma área pequena $\left(4,1 \mathrm{~km}^{2}\right) \mathrm{com}$ alta densidade populacional (36.000 hab./ $\mathrm{km}^{2}$ ) (DEMOGRÁFICO, 2010) e estão localizados em uma área plana, localizado entre uma montanha e uma praia. Tais fatores proporcionam um cenário de alta complexidade para a distribuição urbana, justificando a escolha do local para a realização do teste experimental.

\subsection{Descrição das operações avaliadas neste estudo}

A distribuição postal precede de uma série de atividades internas relacionadas a recebimento, triagem, separação, pesagem, ordenamento e lançamento no sistema, que ocupam grande parte do tempo de serviço do responsável pela distribuição postal, seguidas da atividade de transporte e entrega em si. Entretanto, o presente trabalho tem como objeto de estudo apenas as atividades que têm início e fim nos momentos de saída e retorno do funcionário responsável pela distribuição postal ao Centro de Distribuição (CD).

\subsubsection{Distribuição Tradicional Intermodal (DTI)}

Esta operação é realizada por meio de uma caminhada a pé com apoio de carrinho de mão e bolsa própria, mais a utilização do ônibus convencional e o apoio operacional de um veículo comercial leve com motor à combustão.

Ao sair do $C D$, o carteiro percorre a rota estabelecida de ida, a pé, caso seja próximo, ou de ônibus urbano convencional de linha, até o bolsão de atendimento onde realiza as entregas. Estas são feitas a pé pelo carteiro com apoio de carrinho de mão e bolsa própria. Devido ao limite legal, o peso da bolsa, estabelecido pelo acordo coletivo da categoria, é de $10 \mathrm{~kg}$ para homens e $8 \mathrm{~kg}$ para mulheres. Deste modo, o carteiro tem o apoio de um veículo comercial leve com motor à combustão para realização do transporte do excedente de peso a pontos pré-definidos de sua rota, que são os pontos de apoio para distribuição (PAD's). Ao terminar a entrega de um carregamento, o carteiro se dirige ao PAD para receber mais um carregamento e, a partir daí, tem início uma nova distribuição, que é realizada a pé. Ao término da sua rota, o carteiro retorna ao CD por meio do ônibus urbano convencional de linha, tal como ilustrado na Figura 1.

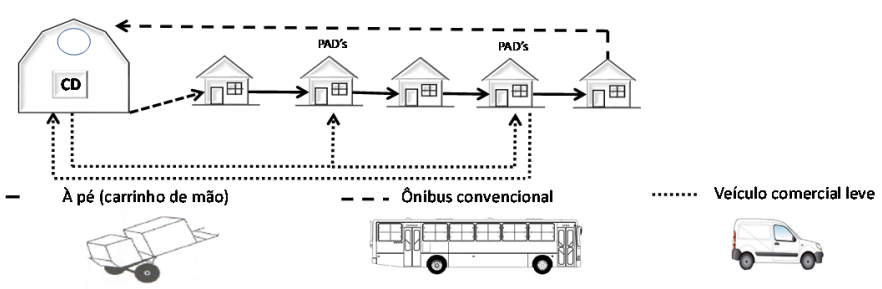

Figura 1: Distribuição Tradicional Intermodal. 


\subsubsection{Distribuição com apoio de Triciclo Elétrico (DTE)}

Esta operação é realizada com o apoio do triciclo elétrico ao longo de todo o percurso, logo, o limite de peso passa a ser a capacidade do triciclo (no caso em questão, a capacidade do triciclo utilizado é de $50 \mathrm{~kg}$ ) e não mais a restrição legal, imposta pelo acordo coletivo da categoria devido ao limite de peso a ser carregado a pé pelo carteiro. Portanto, esta operação dispensa o apoio do veículo comercial leve e da utilização dos PADs.

0 carteiro realiza o deslocamento principal de ida, do CD até o primeiro ponto de atendimento, sobre o triciclo elétrico, pedalando, pedalando simultaneamente com a assistência do motor ou apenas com o uso do motor. Ao chegar ao bolsão de entrega, estaciona o veículo e realiza as entregas nas proximidades a pé, tal como apresentado na Figura 2.

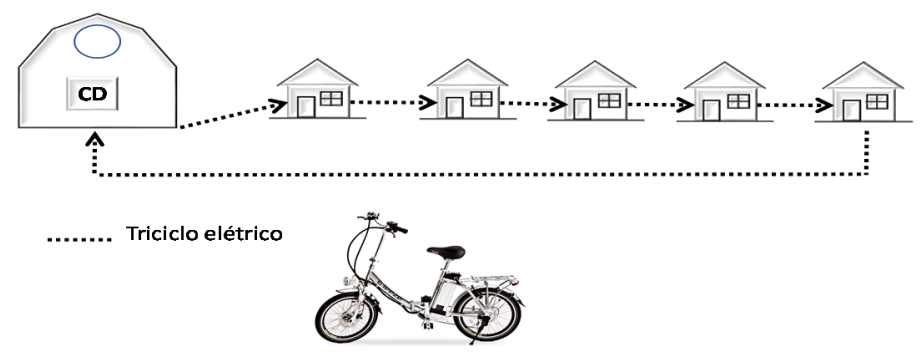

Figura 2: Distribuição com apoio de Triciclo Elétrico.

\subsection{Coleta de dados}

Convencionalmente, o sistema atende somente com estratégia DTI. Porém, foi realizado um experimento de duas semanas com adoção da estratégia DTE. Assim, a coleta de dados foi realizada, de terça a quinta, durante dez dias, sendo cinco dias para cada tipo de operação.

Os dados levantados durante a coleta podem ser agrupados nos seguintes itens: (i) tempo ida, volta e entrega; (ii) distância - ida, volta e entrega; (iii) velocidade média - ida, volta e entrega; (iv) número de clientes atendidos; e (v) frequência cardíaca do carteiro. Para mensurar a frequência cardíaca do carteiro para ambos os tipos de operação, foi utilizado uma unidade do equipamento da marca Garmin, modelo Forerunner 405, com GPS e Medidor Cardíaco, que grava data, hora, latitude, longitude, altitude, distância e batimento cardíaco. Além disso, também foi levantado o consumo médio de combustível e seu respectivo custo para ambos os processos de entrega.

Em seguida, realizou-se o teste de Shapiro-Wilk para verificar a normalidade das amostras, sendo o p-valor encontrado superior a 0,05 (nível de significância adotado) para todos os dados analisados (BUSSAB e MORETTIN, 1987). Deste modo, pode-se afirmar que a amostra segue uma distribuição normal. Assim, a seguir são apresentados os métodos de avaliação adotados neste estudo.

\subsection{Método de avaliação}

Nesta seção, são apresentados o método de avaliação adotado para analisar o impacto da operação sob a perspectiva da sustentabilidade.

\subsubsection{Método de avaliação econômica}

A avaliação econômica para comparação de cada estratégia de distribuição (DTI e DTE) baseouse na análise do nível de serviço e levantamento dos custos de transporte. 
O nível de serviço para cada operação de distribuição foi mensurado com base no número de clientes atendidos, conforme a Equação 1.

Em que,

$$
N S=\frac{\sum c a}{\sum t e}
$$

$N S=$ Nível de Serviço

$c a=$ Número de clientes atendidos;

te $=$ Tempo total de entrega.

Os custos de transporte (soma do custo fixo e variável) foram calculados com base no Manual de Cálculo de Custos e Formação de Preços do Transporte Rodoviário de Cargas (NTC, 2014), conforme Equações 2 e 3.

Em que,

$$
C t_{D T I}=(C c / h * t c)+(C p * n)+(C m / h * t c f)+(C f / k m * d f)
$$

CtDTI $=$ Custo total da DTI

$C c / h=$ Custo do carteiro por hora

$t c=$ Tempo total do ciclo

$C p=$ Custo da passagem de ônibus

$n=$ Número de viagens de ônibus

$\mathrm{Cm} / \mathrm{h}=$ Custo do motorista do veículo comercial leve por hora

$t c f=$ Tempo de ciclo do veículo comercial

$\mathrm{Cf} / \mathrm{km}$ = Custo do furgão por $\mathrm{km}$

$d f=$ Distância percorrida pelo veículo comercial

Em que,

$$
C t_{D T E}=(C c / h * t c)+(C t r / k m * d t r)
$$

CtDTE $=$ Custo total

$C c / h=$ Custo do Carteiro por hora

$t c=$ Tempo total do ciclo

$\mathrm{Ctr} / \mathrm{km}=$ Custo do triciclo por $\mathrm{km}$

$d t r=$ Distância percorrida pelo triciclo em $\mathrm{km}$

É importante salientar que, para cálculo dos custos por km para o veículo comercial leve, foram considerados os custos relativos à depreciação, manutenção, impostos, seguros obrigatórios, combustível e energia elétrica, conforme indicado em NTC (2014).

\subsubsection{Método de avaliação ambiental}

Como a DTE é não emissora de gases poluentes, considerando apenas o uso final, e dispensa à utilização do veículo comercial leve e do ônibus urbano, a avaliação ambiental se baseia na comparação de quanto se deixa de emitir de cada poluente analisado. Os poluentes analisados foram o CO (monóxido de carbono), $\mathrm{N}_{2} \mathrm{O}$ (óxido nitroso), NOx (óxido de nitrogênio), RCHO (aldeídos), NMHC (hidrocarbonetos com a exceção de metano), $\mathrm{CH}_{4}$ (Metano), MP (material particulado), e $\mathrm{CO}_{2}$ (dióxido de carbono).

Para o cálculo das emissões dos Gases de Efeito Estufa (GEE) e dos poluentes atmosféricos (PA) da operação DTI, foi adotado o método bottom-up, de modo que se multiplicou os fatores de emissão de cada gás, obtidos em MMA (2013), pela quilometragem média percorrida, conforme a Equação 4. 
Em que,

$$
E=\left(f e f^{*} d f\right)+((f e o * d o) /(I P K o * d o))
$$

$E=$ Emissão de poluentes atmosféricos da DTI

$f e f=$ Fator de emissão do furgão para cada poluente $(\mathrm{g} / \mathrm{km})$

$f e o=$ Fator de emissão do ônibus para cada poluente $(\mathrm{g} / \mathrm{km})$

$d o=$ Distância percorrida pelo carteiro no ônibus $(\mathrm{km})$

$d f=$ Distância percorrida pelo furgão $(\mathrm{km})$

$I P K o$ = Índice de passageiro por quilômetro do ônibus

O cálculo das emissões de dióxido de carbono $\left(\mathrm{CO}_{2}\right)$ se baseou na multiplicação do fator de emissão, obtido em MMA (2013), pelo consumo médio de combustível para cada veículo de combustão interna utilizado na operação DTI. Ressalta-se que os ônibus são movidos a diesel S10 B7, mistura que contém 7\% em volume de biodiesel e 93\% em volume de óleo diesel de petróleo (MMA, 2013). Ainda, o veículo comercial leve adotado nesta operação é abastecido com gasolina C, mistura que contém $73 \%$ de gasolina automotiva e $27 \%$ de etanol anidro. Os fatores de emissão considerados no estudo são do $2^{\circ}$ Inventário Nacional de Emissões Atmosféricas por Veículos Automotores Rodoviários (MMA, 2013), como apresentado na Tabela 1. Ainda, para estimar o impacto direto dos gases de efeito estufa, foi utilizada a conversão das emissões de $\mathrm{CH}_{4}$ e $\mathrm{N}_{2} \mathrm{O}$ para o equivalente de $\mathrm{CO}_{2}$ (cálculo do $\mathrm{CO}_{2}$ equivalente), considerando as taxas de 21 GWP e 310 GWP, respectivamente.

Tabela1: Fatores de emissão considerados no estudo.

\begin{tabular}{|c|c|c|c|c|c|c|c|c|c|}
\hline Ano/Modelo & Combustivel & $\mathrm{CO}^{(1)}$ & $\mathrm{NO}_{\mathrm{x}}(1)$ & $\mathrm{RCHO}^{(1)}$ & $\mathrm{NMHC}_{\text {escap }}{ }^{(1)}$ & $\mathrm{CH}_{4}{ }^{(1)}$ & $M P^{(1)}$ & $\mathrm{N}_{2} \mathrm{O}^{(1)}$ & $\mathrm{CO}_{2}{ }^{(2)}$ \\
\hline 2013 & Flex - Gasolina C & 0,25 & 0,0030 & 0,0017 & 0,014 & 0,026 & 0,0011 & 0,026 & 2,0267 \\
\hline P6 e P7 PROCONVE 2012 & Diesel & 0,44 & 2,103 & - & 0,033 & 0,06 & 0,0200 & 0,03 & 2,5909 \\
\hline
\end{tabular}

Nota: (1) g/km; (2) kg/l, destaca-se que os valores apresentados foram obtidos por meio da média ponderada da proporção de combustíveis na mistura. Fonte: MMA (2013)

Foram levantados junto ao Sindicato das Empresas de Ônibus da Cidade do Rio de Janeiro (Rio Ônibus) dados das operações do setor, viabilizando as estimativas do IPK $(1,67)$ e o rendimento energético $(2,59 \mathrm{~km} / \mathrm{l})$ dos ônibus municipais. Ademais, o rendimento do veículo comercial leve foi realizado a partir dos dados levantados durante a operação, estimando um rendimento médio de $4,56 \mathrm{~km} / \mathrm{l}$.

\subsubsection{Método de avaliação social}

O esforço realizado pelo carteiro em cada uma das operações de distribuição pode ser avaliado pelo acompanhamento do batimento cardíaco e medido pela Frequência Cardíaca (FC) máxima, estimada com base na idade do indivíduo por meio da Equação 5, proposta por Arena et al., (2016).

Sendo,

$$
F C M=209.3-0.72 *(i)
$$

$F C M=$ Frequência Cardíaca Máxima (batimentos por minuto);

$i=$ idade (anos).

Esse índice representativo da carga de trabalho realizada por motoristas também é utilizado em estudos semelhantes, como Hu et al. (2018). É importante salientar que, inerente a todas equações para estimar a FC máxima de um indivíduo, há um erro padrão, visto que a FC máxima 
é uma variável fisiológica difícil de se determinar devido à complexidade das variáveis cardíacas em geral (assim como a relação entre FC máxima e idade) (Froelicher et al, 1998; Almeida, 2014).

Conforme o Americam College of Sports Medicine (ACSM, 1998), dividiu-se a frequência dos batimentos por minuto em seis faixas percentuais em relação a FC máxima: (i) 40 - 50\%, Repouso; (ii) 50 - 60\%, Zona de Manutenção; (iii) 60 - 70\%, Zona Aeróbia Lipolítica; (iv) 70 - 80\%, Zona do Limiar Aeróbio; (v) 80 - 90\%, Zona Mista e (vii) 90 - 100\%, Zona de Esforço Máximo.). A medida de comparação é dada pelo tempo percentual que a frequência medida permanece em cada faixa de esforço durante a realização da atividade.

\section{RESULTADOS E ANÁLISES}

A Tabela 2 apresenta o detalhamento dos itens considerados para composição dos custos totais da rota analisada para cada tipo de distribuição (DTI e DTE). Assim, com base no método de avaliação econômica apresentado na seção 3.3.1, foi possível calcular os custos fixos e variáveis para cada operação, conforme apresentado na Tabela 3. Desta forma, verificou-se que o custo total diário das rotas analisadas para as operações de distribuição DTI e DTE foi de R\$38,31 e $\mathrm{R} \$ 27,63$, respectivamente, sendo possível identificar uma economia dos custos de $27,86 \%$.

Tabela 2: Detalhamento dos itens considerados para composição dos custos totais por tipo de operação (DTI e DTE).

\begin{tabular}{|c|c|c|c|}
\hline Especificações da operação & Und & DTI & DTE \\
\hline Quantidade de dias mês considerados & und & 26 & 26 \\
\hline Quilometragem média percorrida na rota & $\mathrm{km}$ & 5,01 & 10,74 \\
\hline $\mathrm{n}$ o de viagens por dia & und & 2 & 1 \\
\hline no médio de passagens de ônibus & und & 2,40 & - \\
\hline Valor da passagem de ônibus em $\mathrm{R} \$$ & $\mathrm{R} \$$ & 3,00 & - \\
\hline no de carteiros atendidos na rota & und & 10 & 1 \\
\hline
\end{tabular}

Tabela 3: Especificação dos custos fixos e variáveis, considerados nas operações DTI e DTE.

\begin{tabular}{|c|c|c|c|}
\hline Custos Fixos Mensais & Und & VCL & Triciclo \\
\hline Remuneração de capital & $\mathrm{R} \$$ & 309,80 & 33,12 \\
\hline Salário do motorista + encargos & $\mathrm{R} \$$ & $2.636,10$ & - \\
\hline Salário do carteiro + encargos em rota & $\mathrm{R} \$$ & 590,11 & 459,51 \\
\hline Salário de oficina & $\mathrm{R} \$$ & 425,65 & 44,34 \\
\hline Reposição do veículo & $\mathrm{R} \$$ & 161,63 & 47,20 \\
\hline Licenciamento & $\mathrm{R} \$$ & 120,55 & - \\
\hline Seguro do casco do veículo & $\mathrm{R} \$$ & 162,81 & - \\
\hline Seguro de responsabilidade civil facultativa & $\mathrm{R} \$$ & 94,92 & - \\
\hline Total: & $\mathrm{R} \$$ & $4.501,56$ & 584,17 \\
\hline Custos Variáveis: & Und & VCL & Triciclo \\
\hline Peças, acessórios e materiais de manutenção & $\mathrm{R} \$ / \mathrm{km}$ & 0,7100 & 0,1973 \\
\hline Combustiveis & $\mathrm{R} \$ / \mathrm{km}$ & 0,7348 & 0,0100 \\
\hline Lubrificantes & $\mathrm{R} \$ / \mathrm{km}$ & 0,0153 & - \\
\hline Reposição de Baterias & $\mathrm{R} \$ / \mathrm{km}$ & - & 0,0377 \\
\hline Lavagem e lubrificação & $\mathrm{R} \$ / \mathrm{km}$ & 0,2857 & 0,1432 \\
\hline Pneus & $\mathrm{R} \$ / \mathrm{km}$ & 0,0327 & 0,0309 \\
\hline Total: & $\mathrm{R} \$ / \mathrm{km}$ & 1,7786 & 0,4192 \\
\hline
\end{tabular}

Quanto à avaliação ambiental, foram estimadas apenas as emissões da operação DTI, uma vez que esta utiliza veículos ciclo Otto e ciclo Diesel, enquanto a operação DTE utiliza veículo elétrico, portanto pode ser considerada uma emissão final nula. Logo, obtiveram-se as reduções 
mensais de poluentes por rota de 10,11 g de CO; 32,78 g de NOx; 0,81g de $\mathrm{N}_{2} \mathrm{O} ; 0,02 \mathrm{~g}$ de RCHO; 0,7 g de NMHC; 1,27 g de $\mathrm{CH}_{4} ; 0,33 \mathrm{~g}$ de MP e 20,73 $\mathrm{kg}$ de $\mathrm{CO}_{2}$. Destaca-se que, considerando apenas as emissões de GEE, tem-se uma emissão de 21,01 kg de $\mathrm{CO}_{2 \text { eq. }}$.

No tocante ao aspecto social, a Tabela 4 evidencia a comparação entre a FC coletada nas operações DTI e DTE. Realizou-se a medição em um funcionário de 34 anos, com 82 kg e FC máxima (estimada pela Equação 5) de 184 bpm.

Tabela 3: Faixas de FC medidas nas operações DTI e DTE

\begin{tabular}{|c|c|c|c|c|c|c|}
\hline Faixas de FC & & DTI média (\%) & $\begin{array}{l}\text { Desvio } \\
\text { Padrão }\end{array}$ & DTE média (\%) & $\begin{array}{l}\text { Desvio } \\
\text { Padrão }\end{array}$ & $\begin{array}{l}\text { Variação \% } \\
\text { DTI x DTE }\end{array}$ \\
\hline$<50 \%$ & $0-91$ bpm & 23,93 & 21,93 & 9,29 & 6,12 & $-61,17$ \\
\hline $50-60 \%$ & $92-109$ bpm & 52,42 & 15,84 & 84,83 & 2,59 & 61,81 \\
\hline $60-70 \%$ & $110-128 \mathrm{bpm}$ & 22,01 & 22,09 & 5,88 & 4,85 & $-73,27$ \\
\hline $70-80 \%$ & $129-146$ bpm & 1,64 & 3,28 & 0,00 & 0,00 & - \\
\hline $80-90 \%$ & $147-165$ bpm & 0,00 & 0,00 & 0,00 & 0,00 & - \\
\hline $90-100 \%$ & $166-184$ bpm & 0,00 & 0,00 & 0,00 & 0,00 & - \\
\hline
\end{tabular}

Observa-se a ocorrência de batimentos na Zona do Limiar Aeróbio (70 a 80\% da FC máxima) apenas na operação DTI. Na outra vertente, em cerca de $84 \%$ do tempo total de entrega da operação DTE, a frequência cardíaca do entregador ficou na Zona de Manutenção (entre 50 e 60\% da FC máxima), sendo 61\% maior do que na operação DTI. Os dados inferem que, na operação de DTI, há uma maior variação da FC durante toda a operação de entrega (com maior pico do esforço), enquanto na operação DTE apresentou uma tendência a um platô, com FC constante durante toda a operação.

Com relação ao nível de serviço, observou-se um aumento de $26 \%$ da produtividade, se comparada à operação DTE em relação à operação DTI. Verificou-se que a operação DTE, além de consumir menos tempo, foi capaz de realizar um maior número de entregas, atendendo mais clientes na rota analisada, conforme pode ser observado na Figura 3.

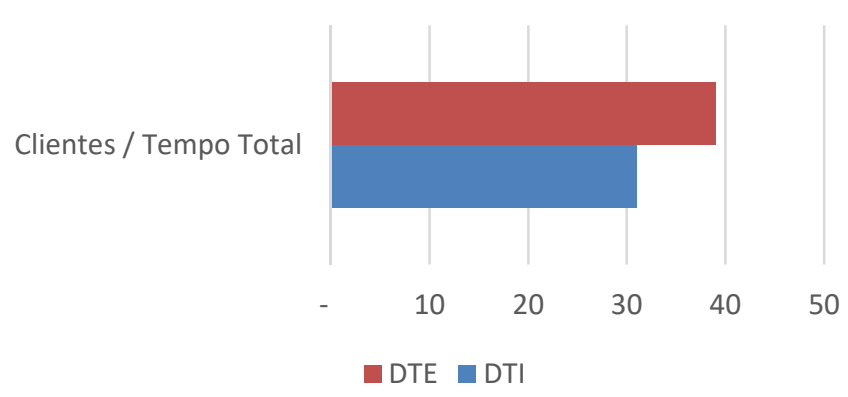

Figura 3: Comparação do nível de serviço

Assim, os resultados encontrados neste estudo apontam que a adoção triciclo elétrico para a distribuição postal, na cidade do Rio de Janeiro, está alinhado com a necessidade de eliminar as externalidades negativas produzidas pela atividade do transporte, sobretudo por causa do grande consumo de energia de origem fóssil, apontado por Rothengatter et al. (2003). Além disso, como os resultados apontaram redução de custos e melhoria do nível de serviço, mostram-se também coerente com o estudo de Foltyński (2014), que aponta a eficiência da alternativa de adotar veículos elétricos para o transporte de carga em áreas urbanas. 
A operação DTE também trouxe vantagens sob o ponto de vista social, uma vez que se observou que, na operação DTE, a frequência cardíaca do entregador ficou na Zona de Manutenção (entre 50 e $60 \%$ da FC máxima) em um intervalo de tempo 61\% maior do que na operação DTI, trazendo assim vantagens para a saúde do trabalhador. Este resultado está alinhado ao estudo de Hinde e Dixon (2005), que aponta a contribuição do uso de triciclos elétricos na manutenção da saúde das pessoas, na medida em que possibilita a realização de exercício físico para os trabalhadores.

Desta forma, a análise dos resultados encontrados permite constatar que se justifica a inserção dos triciclos elétricos na operação de distribuição postal, em área urbana, no segmento de distribuição postal, na cidade do Rio de Janeiro, tanto do ponto de vista ambiental, devido às emissões evitadas, quanto do ponto de vista econômico, devido à redução do custo de distribuição, e do ponto de vista social, por meio da análise da FC do trabalhador. Além disso, verificouse também uma melhoria quanto ao nível de serviço, tendo em vista a redução de tempo alcançado e o aumento do número de clientes atendidos.

Ainda, é importante destacar que, para o caso da inserção dos triciclos elétricos na operação de para a distribuição postal do Rio de Janeiro, não seria necessária a adoção de incentivos tributários por parte do poder público. Mesmo considerando a aquisição dos veículos elétricos pela empresa, a análise econômica ainda apresentou uma redução no custo total de transportes da operação DTE em relação à DTI.

Por fim, constatou-se que a utilização do triciclo elétrico como alternativa para a distribuição postal em last mile foi capaz de promover benefícios econômicos, ambientais, sociais e melhorar o nível de serviço da empresa pública de distribuição postal na cidade do Rio de Janeiro.

\section{CONCLUSÕES, LIMITAÇÕES E SUGESTÕES}

O objetivo deste trabalho consiste em avaliar a sustentabilidade por meio da utilização do triciclo elétrico na distribuição postal, em área urbana, na cidade do Rio de Janeiro. Os métodos de avaliação adotados, bem como a técnica de coleta de dados e da observação não estruturada, se mostraram adequados ao estudo realizado.

Identificou-se uma redução de $27,86 \%$ do custo total de transportes por rota devido à adoção do triciclo elétrico na distribuição postal urbana. Esta prática também implica na redução da emissão de poluentes atmosféricos e GEE. Obtiveram-se reduções mensais de poluentes atmosféricos por rota de 10,11 g de CO; 32,78 g de NOx; 0,02 g de RCHO; 0,7 g de NMHC; e 0,33 g de MP. As reduções mensais de GEE por rota foram de 20,73 $\mathrm{kg}$ de $\mathrm{CO}_{2}, 0,81 \mathrm{~g}$ de $\mathrm{N}_{2} \mathrm{O}$; e 1 ,27 g de $\mathrm{CH}_{4}$, o que equivale a uma emissão de $21,01 \mathrm{~kg}$ de $\mathrm{CO}_{2}$ eq.

Com relação aos aspectos sociais, observou-se que, na operação DTE, a FC apresentou uma tendência a um platô, sendo constante durante toda a operação, enquanto, na operação de DTI, verificou-se uma maior variação da FC durante toda a operação de entrega (com maior pico do esforço). Deste modo, pode-se inferir que a adoção dos triciclos elétricos trouxe vantagens para a saúde do trabalhador, o que implica em vantagens de cunho social.

Por fim, no tocante ao nível de serviço oferecido pela empresa, verificou-se um aumento de $26 \%$ da produtividade, se comparada à operação DTE em relação à operação DTI. Portanto, a análise, neste estudo, mostrou que a alternativa do triciclo elétrico é viável tanto nos aspectos econômico, ambiental e social, quanto na manutenção do nível de serviço da empresa.

Enfim, verificou-se que a utilização do triciclo elétrico como alternativa para a distribuição 
postal em last mile foi capaz de promover benefícios econômicos, ambientais, sociais e melhorar o nível de serviço da empresa pública de distribuição postal na cidade do Rio de Janeiro.

\section{REFERÊNCIAS}

ACSM (1998) Position Stand. The recommended quantity and quality of exercise for developing and maintaining cardiorespiratory and muscular fitness, and flexibility in healthy adults. Med. Sci. Sports Exerc. 30, p. 975-991, 1998

Allen, J.; M. Browne (2008). Review of Survey Techniques Used in Urban Freight Studies. Report produced as part of the Green Logistics Project: Work Module 9 (Urban Freight Transport). London, U.K. Disponível em: http://www.greenlogistics.org.

Almeida, M. B. (2007). Frequência Cardíaca e Exercício: Uma interpretação baseada em evidências. Rev. Bras. Cineantropom. Desempenho Hum., v. 9, n. 2, p. 196-202.

Arena, R.; J. Myers e L. A. Kaminsky. (2016). Revisiting age-predicted maximal heart rate: Can it be used as a valid measure of effort? Am. Heart J. v. 173, p. 49-56.

Bradley, M. J. (2007) Comparison of energy use \& CO2 emissions from different transportation modes. Washington DC; 2007.

Bussab, W. O. e P. A. Morettin (1987) Estatística Básica - 4⿳a Ed, Atual Editora.

Cherry, C. R. et al. (2009) Comparative Environmental Impacts of Electric Bikes in China. Transportation Research, v. 14, p. 281- 290

DECOPE (2014). Manual de Cálculo de Custos e Formação de Preços do Transporte Rodoviário de Cargas. Departamento de Custos Operacionais, Estudos Técnicos e Econômicos. São Paulo.

IBGE (s/d) Censo Demográfico. Disponível em: http://www. ibge. gov. br. Acesso em, v. 3, 2010.

Dutra; N. G. S. (2004) Enfoque de "City Logistics" na Distribuição Urbana de Encomendas. Tese (Doutorado em Engenharia de Produção). Programa de Pós-Graduação em Engenharia de Produção, Centro Tecnológico da UFSC. Florianópolis, Santa Catarina, Brasil.

Foltyński, M. (2014) Electric fleets in urban logistics. Procedia Social and Behavioral Sciences, v. 151, p. 48-5 9. DOI: 10.1016/j.sbspro.2014.10.007

Froelicher, V. F.; J. Myers; W. P. Follansbee e A. J. Labovitz. (1998). Exercício e o coração. 3 ed. Rio de Janeiro: Revinter.

Hu, J e R. Wang. (2018) Classification of driving workload affected by highway alignment conditions based on classification and regression tree algorithm. Traffic Injury Prevention, v. 19, n. 2, p. 214-218.

Leonardi, J.; M. Browne e J. Allen. (2012) Before-after assessment of a logistics trial with clean urban freight vehicles: A case study in London. Procedia - Social and Behavioral Sciences, v. 39, p. 146 - 157.

MCT - Ministério da Ciência, Tecnologia e Inovação (2010). Segundo Inventário Brasileiro de Emissões Antrópicas de Gases de Efeito Estufa. Relatórios de Referência: Emissões de Gases de Efeito Estufa no Transporte Rodoviário.

MMA (2013). Inventário Nacional de Emissões Atmosféricas por Veículos Automotores Rodoviários 2013: Ano-base 2012. Ministério do Meio Ambiente, Brasília, DF.

ONU. (2013). Mais de 70 da população mundial viverá em cidades até 2050. Disponível em: http://www.onu.org.br/onu-maisde-70-da-populacao-mundial-vivera-em-cidades-ate-2050/. Acesso em 08 de Outubro de 2015.

Paudel, A. M. e P. Kreutzmann, P. (2015) Design and performance analysis of a hybrid solar tricycle for a sustainable local commute. Renewable and Sustainable Energy Reviews, v. 41, p. 473-482. Disponível em: DOI: 10.1016/j.rser.2014.08.078

Peres, L. A. P.; A. P. F. Ferreira; A. R. Krempser e T. S. Ferreira (2012) Benefícios Energéticos e Ambientais da Utilização de Triciclos Elétricos em Centros Urbanos no Brasil Cleiton Magalhães. XIV CBE Congresso Brasileiro de Energia Rio de Janeiro. Disponível em: http://www.gruve.eng.uerj.br/download/BeneficiosdaUtilizacaodeTriciclosEletricosFINAL. /. Acesso em 28 de Janeiro de 2016.

Rothengatter, W e D. A. Hensher (2003) Button KJ. Environmental concepts - physical and economic. Handbook of transportation and the environment. 1st ed. Amsterdam: Netherlands: Elsevier Ltd.; 2003, p. 827.

Sivak, M. e Schoettle, B (2012): Eco-driving: Strategic, tactical, and operational decisions of the driver that influence vehicle fuel economy. Transport Policy, v. 22, p. 96-99.

Suksri, J. e Raicu, R. (2012) Developing a conceptual framework for the evaluation of urban freight distribution initiatives. Procedia - Social and Behavioral Sciences, v. 39, p. 321 - 332.

Todd, G. e Gorham, R. (2006) Sustainable urban transport: Four innovative directions. Technology in Society, v. 28, p. 261-273. 